\title{
Rho-kinase regulates negatively the epidermal growth factor-stimulated colon cancer cell proliferation
}

\author{
MASANORI NAKASHIMA $^{1}$, SEIJI ADACHI ${ }^{1,2}$, ICHIRO YASUDA ${ }^{1}$, \\ TAKAHIRO YAMAUCHI ${ }^{1}$, OSAMU KOZAWA ${ }^{2}$ and HISATAKA MORIWAKI ${ }^{1}$ \\ Departments of ${ }^{1}$ Gastroenterology and ${ }^{2}$ Pharmacology, Gifu University \\ Graduate School of Medicine, Gifu 501-1194, Japan
}

Received October 16, 2009; Accepted December 3, 2009

DOI: 10.3892/ijo_00000533

\begin{abstract}
It has been reported that Rho and Rho-kinase are involved in actin cytoskeleton organization and associated with carcinogenesis and progression of human cancers. However, the mechanism how the Rho/Rho-kinase pathway is involved in cell cycle progression has not been precisely characterized. In this study, we investigated the role of Rhokinase in epidermal growth factor (EGF) signaling in SW480 colon cancer cells. We found that Y27632, a Rho-kinase inhibitor, dose-dependently induced cell proliferation in these cells. The blockade of EGF stimulation utilizing anti-EGF receptor neutralizing antibodies significantly suppressed cell growth, suggesting that EGF stimulation plays an important role in cell proliferation in SW480 cells. We also found that EGF induced Rho-kinase activation. Interestingly, EGFinduced phosphorylation of both Akt and glycogen synthase kinase-3ß (GSK-3ß), but not p44/p42 mitogen-activated protein (MAP) kinase, were dose-dependently enhanced when the cells were pretreated with Y27632 or fasudil, another Rho-kinase inhibitor. Moreover, whereas EGF increased the phosphorylation of retinoblastoma tumor suppressor protein as well as cyclin D1 protein expression level, pretreatment with Y27632 accelerated them. Taken together, our results suggest that Rho-kinase regulates negatively EGF-induced cell proliferation upstream of Akt/GSK-3B in colon cancer cells.
\end{abstract}

\section{Introduction}

GTPases of the Ras and Rho superfamily act as molecular switches to control a wide range of essential biological pathways in all eukaryotic cells (1). In their active, GTP-bound state, Ras and Rho GTPases interact with target proteins to promote a cellular response (1). Among the super-family, Rho regulates the formation of contractile actin-myosin

Correspondence to: Dr Seiji Adachi, Department of Pharmacology, Gifu University Graduate School of Medicine, Gifu 501-1194, Japan

E-mail: seijiadachi0123@gmail.com

Key words: Rho-kinase, EGF, cell growth, colon cancer filaments, which form stress fibers, and maintains focal adhesions at the rear of the cell (2). Increasing evidence implicates Rho GTPases as major players in the regulation of a variety of cellular processes in addition to contraction (3). Rho GTPases reportedly contribute to the regulation of cell cycle progression, cell motility and invasive phenotypes (3). Although overexpression of Rho has been linked to progression of human cancers (4), the roles of the Rho GTPases and their downstream underlying cancer progression and invasion remain controversial.

Rho-kinase is one of the various targets to Rho (5). Accumulating evidence indicates that the Rho/Rho-kinase pathway plays an important role in various cellular functions such as vascular smooth muscle cell contraction, cell migration and cell proliferation (3). Action of the Rho/Rho-kinase pathway takes part in cancer progression by regulating actin cytoskeleton reorganization. Since a specific Rho-kinase inhibitor was found to suppress tumor growth and metastasis (6), it is generally recognized that the Rho/Rho-kinase pathway may become a molecular target for prevention of cancer invasion and metastasis.

On the other hand, the ability of cancer cells for survival is dependent largely on the epidermal growth factor (EGF) (7). Through its binding to cell surface EGF receptor (EGFR), EGF activates an extensive network of signal transduction pathways that include activation of the Ras/p44/p42 mitogen-activated protein (MAP) kinase and phosphatidyl inositol-3-kinase (PI3K)/Akt pathways (7). Glycogen synthase kinase (GSK)-3 is a critical downstream element of the PI3K/Akt pathway, and its activity can be inhibited by Akt-mediated phosphorylation of GSK-3 $\alpha$ at Ser2 1 and GSK-3ß at Ser9 $(8,9)$. Unlike most protein kinases, GSK-3ß is typically active in unstimulated cells (10). Thus, GSK-3ß phosphorylates Thr286 of cyclin D1 (11) and phosphorylated cyclin D1 is exported from the nucleus and degraded by the $26 \mathrm{~S}$ proteasome system after ubiquitination (12). It has also been demonstrated that activation of PI3K and Akt-mediated phosphorylation of GSK-3ß negatively regulate its activity, thus promoting increased cyclin D1 protein levels (11). Furthermore, cyclin D1 complex with cyclin-dependent kinase (CDK) 4 and CDK6 stimulate their kinase activities, which in turn cause the phosphorylation and inactivation of the retinoblastoma tumor-suppressor protein ( $\mathrm{Rb})(11)$, directing toward cell proliferation. However, the 
relationship between Rho-kinase and EGF signaling in cancer cells has not been precisely clarified.

In the present study, we investigated the role of Rho-kinase in EGF-induced colon cancer cell progression. Interestingly, we found for the first time that Rho-kinase plays a negative role in EGF-stimulated proliferation of colon cancer cells.

\section{Materials and methods}

Materials. Y27632 was obtained from CalbiochemNovabiochem Co. (La Jolla, CA). EGF and hydroxyfasudil (fasudil) were purchased from Sigma Chemical Co. (St. Louis, MO). Anti-phospho-specific myosin phosphatase targeting subunit-1 (MYPT-1) (Thr850) antibodies were purchased from Upstate (Lake Placid, NY). Anti-MYPT-1 antibodies were purchased from Santa Cruz Biotechnology, Inc. (Santa Cruz, CA). Antibodies against phospho-specific p44/p42 MAP kinase, p44/p42 MAP kinase, phosphospecific Akt, Akt, phospho-specific GSK-3B, GSK-3ß, cyclin D1, phospho-specific Rb and phospho-specific myosin regulatory light chain (MLC) were purchased from Cell Signaling Inc. (Beverly, MA). Anti-EGFR-neutralizing antibodies were purchased from Millipore (Temecula, CA). ECL Western blot detection system was purchased from Amersham Pharmacia Biotech (Buckinghamshire, UK). Other materials and chemicals were obtained from commercial sources. Y27632 and fasudil were dissolved in dimethyl sulfoxide (DMSO). The maximum concentration of DMSO was $0.1 \%$, which did not affect the assay for Western blot analysis.

Cell culture and chemicals. Unless indicated otherwise SW480 human colon cancer cells were grown in Dulbecco's modified Eagle's medium (DMEM) (Invitrogen, San Diego, CA), containing $10 \%$ fetal calf serum (FCS). Before the experiments, they were incubated in serum-free medium for an additional $24 \mathrm{~h}$ as described previously (13).

Cell proliferation assay. Cell proliferation assay was performed using cell proliferation ELISA (5-bromo-deoxyuridine: BrdU) and 3-(4,5-dimethylthiazol-2-yl)-2,5-diphenyltetrazolium bromide (MTT) cell proliferation kit I (Roche Diagnostics Co., Indianapolis, IN), according to the instructions of the manufacturer, respectively. In brief, SW480 cells were plated onto 96 -well plates $\left(3 \times 10^{3}\right.$ cells $/$ well) and $24 \mathrm{~h}$ later, the cells were treated with the indicated doses $(0-10 \mu \mathrm{M})$ of $\mathrm{Y} 27632$ for $48 \mathrm{~h}$ in DMEM containing $10 \%$ FCS. The medium and agents were not changed during this time period. In Fig. 1C, the attached cells were treated with $0.5 \mu \mathrm{g} / \mathrm{ml}$ of anti-EGFR-neutralizing antibodies or normal mouse-IgG for 5 days under medium containing 3\% FCS and then the remaining cells were counted by MTT cell proliferation kit I. All assays were done in triplicate.

Western blot analysis. Western blot analysis was performed as described previously (13). The cultured cells were pretreated with various doses of Y27632 or fasudil for $60 \mathrm{~min}$, and then stimulated by $30 \mathrm{ng} / \mathrm{ml}$ of EGF for the indicated periods. Protein extracts were examined by Western blot analysis. The protein $(12 \mu \mathrm{g})$ was fractionated and transferred
(A)

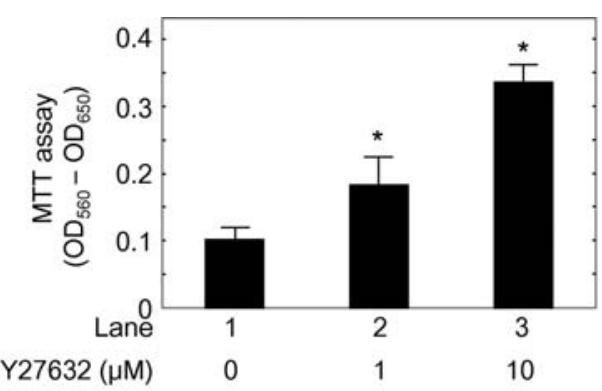

(B)

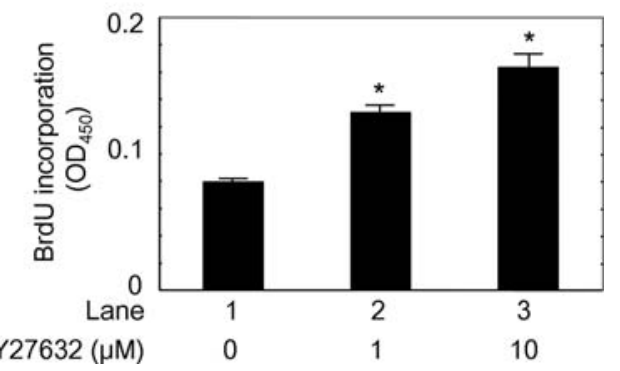

(C)

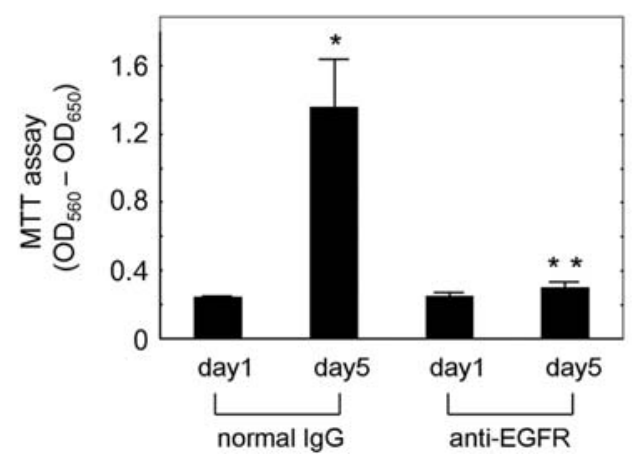

Figure 1. Effects of Y27632 on anti-EGFR-neutralizing antibodies in SW480 colon cancer cell proliferation. (A) Cell viability assay was performed using the MTT cell proliferation kit I. Results are expressed as the absorbance (OD $560 \mathrm{~nm}-\mathrm{OD} 750 \mathrm{~nm}$ ). (B) The measurement of BrdU incorporation during DNA synthesis was also performed using cell proliferation ELISA (BrdU). Results are expressed as the absorbance (OD $450 \mathrm{~nm}$ ). Bars designate SD of triplicate assays. (C) The attached cells were treated with $0.5 \mu \mathrm{g} / \mathrm{ml}$ of anti-EGFR-neutralizing antibodies or normal mouse-IgG for 5 days under medium containing 3\% FCS and then the remaining cells were counted by MTT cell proliferation kit I. All assays were done in triplicate.

onto an Immuno-Blot PVDF Membrane (Bio-Rad, Hercules, CA). Membranes were blocked with $5 \%$ fat-free dry milk in phosphate-buffered saline (PBS) containing $0.1 \%$ Tween-20 (PBS-T) for $30 \mathrm{~min}$ before incubation with the indicated primary antibodies. Peroxidase-labeled antibodies raised in goat against rabbit IgG were used as second antibodies. Peroxidase activity on the membrane was visualized on Xray film by means of the ECL Western blot detection system.

Immunofluorescence microscopy studies. Immunofluorescence microscopy studies were performed as described previously (14). The cells grown on coverslip-bottom dishes were treated with or without $\mathrm{Y} 27632(10 \mu \mathrm{M})$ for $1 \mathrm{~h}$ at $37^{\circ} \mathrm{C}$, followed by exposure to $30 \mathrm{ng} / \mathrm{ml}$ of EGF for $15 \mathrm{~min}$ at $37^{\circ} \mathrm{C}$. They were then fixed with $4 \%$ paraformaldehyde for $10 \mathrm{~min}$ on ice and then exposed to $0.1 \%$ Triton X-100 for $10 \mathrm{~min}$ to 
(A)
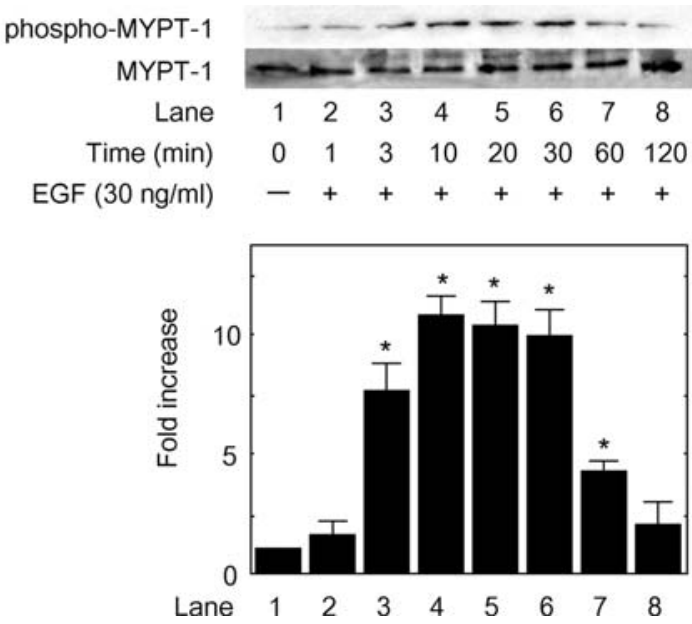

(C) phospho-MYPT-1
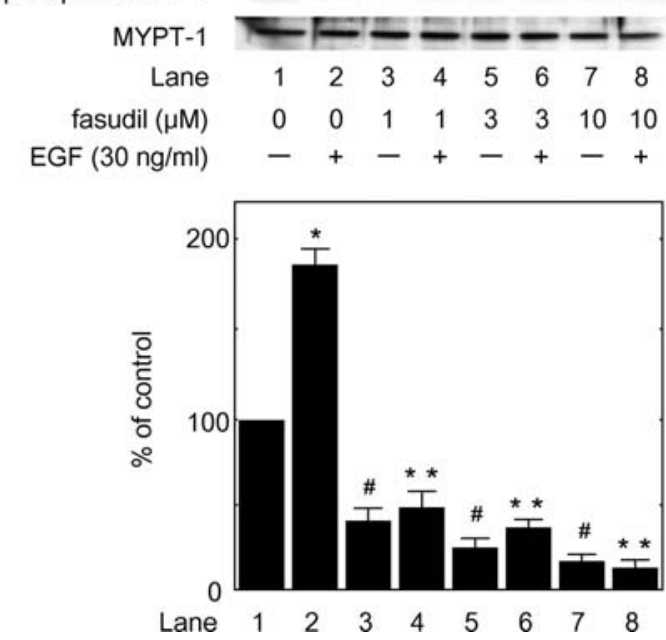

(B) phospho-MYPT-1

MYPT-1

Lane

Y27632 ( $\mu \mathrm{M})$

EGF $(30 \mathrm{ng} / \mathrm{ml})$
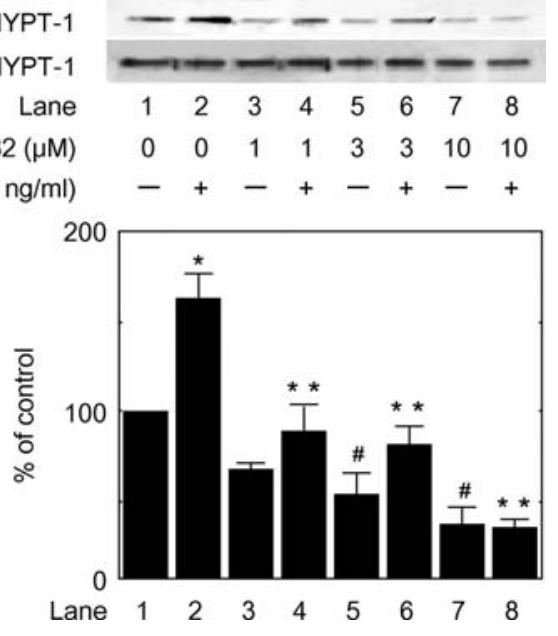

(D)

DMSO
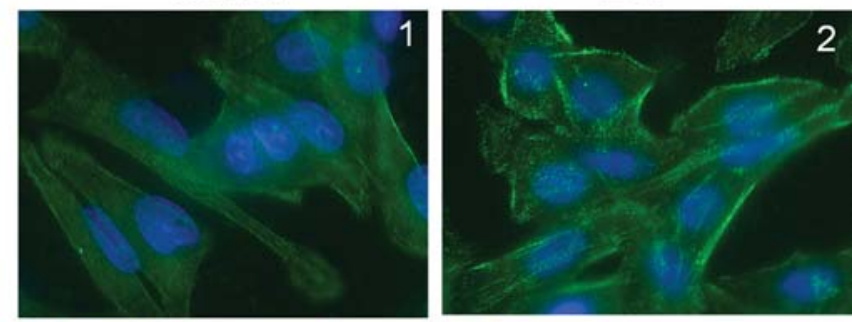

3

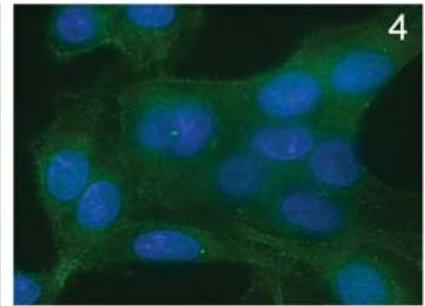

Figure 2. Effects of Rho-kinase inhibitors on EGF-induced phosphorylation of MYPT-1 in SW480 colon cancer cells. (A) SW480 cells were stimulated with $30 \mathrm{ng} / \mathrm{ml}$ EGF for the indicated periods. The lower bar graph shows the quantification data for the relative levels of phospho-MYPT-1, after normalization with respect to total MYPT-1, as determined by densitometry. The asterisks indicate significant increase (" $<<0.05$, compared with the control). (B and C) SW480 cells were pretreated with various doses of Y27632 (B) or fasudil (C) for $60 \mathrm{~min}$, and then stimulated with $30 \mathrm{ng} / \mathrm{ml} \mathrm{EGF}$ or vehicle for $20 \mathrm{~min}$. The extracts of cells were subjected to SDS-PAGE with subsequent Western blot analysis with antibodies against phospho-specific MYPT-1 or MYPT-1. The lower bar graph shows the quantification data for the relative levels of phospho-MYPT-1, after normalization with respect to total MYPT-1, as determined by densitometry. $\left({ }^{*}\right)$ indicates significant increase $(\mathrm{p}<0.05)$, as compared with the control (lane 1), respectively. $\left({ }^{* *}\right)$ indicates significant decrease $(\mathrm{p}<0.05)$, as compared with the control (lane 2), respectively. ( $\left.{ }^{\#}\right)$ indicates significant decrease ( $\left.\mathrm{p}<0.05\right)$, as compared with the control (lane 1), respectively. (D) SW480 cells grown on coverslip-bottom dishes were treated with or without Y27632 $(10 \mu \mathrm{M})$ for $60 \mathrm{~min}$ at $37^{\circ} \mathrm{C}$, followed by exposure to $30 \mathrm{ng} / \mathrm{ml}$ of EGF for $20 \mathrm{~min}$ at $37^{\circ} \mathrm{C}$. After fixation, they were then exposed to anti-phospho MLC antibodies, followed by exposure to Alexa Fluor 488-conjugated goat anti-rabbit IgG antibodies and DAPI for $1 \mathrm{~h}$. The cells were then examined by fluorescence microscopy.

permeabilize the cell membrane. They were then exposed to anti-phospho MLC antibodies, followed by exposure to Alexa Fluor $488^{\circledR}$ conjugated goat anti-rabbit IgG antibodies and 4',6-diamidino-2-phenylindole (DAPI) (Wako, Tokyo, Japan) for $1 \mathrm{~h}$. The cells were then examined by fluorescence microscopy, BIOREVO (BZ-9000) (Keyence, Tokyo, Japan) according to the manufacturer's protocol.

Densitometric analysis. The densitometric analysis was performed using scanner and image analysis software (image J ver. 1.32). The background subtracted signal intensity of each protein signal was normalized by the respective control signal. The data were obtained from at least three independent experiments.

\section{Results}

Effect of Y27632 on cell proliferation in SW480 colon cancer cells. In order to clarify whether Rho-kinase is involved in colon cancer cell proliferation, we first performed cell proliferation assay using MTT and BrdU in SW480 cells grown in medium containing 10\% FCS. We used Y27632 as a specific Rho-kinase inhibitor (15) and found in MTT cell proliferation assay that the cell growth was dose-dependently enhanced when the cells were treated with Y27632 (Fig. 1A). As well, BrdU incorporation was also increased in Y27632treated SW480 cells (Fig. 1B). Therefore, it is likely that Rhokinase plays a suppressive role in SW480 cell proliferation.

Effect of anti-EGFR-neutralizing antibodies on cell proliferation in SW480 colon cancer cells. We next examined the 
(A)
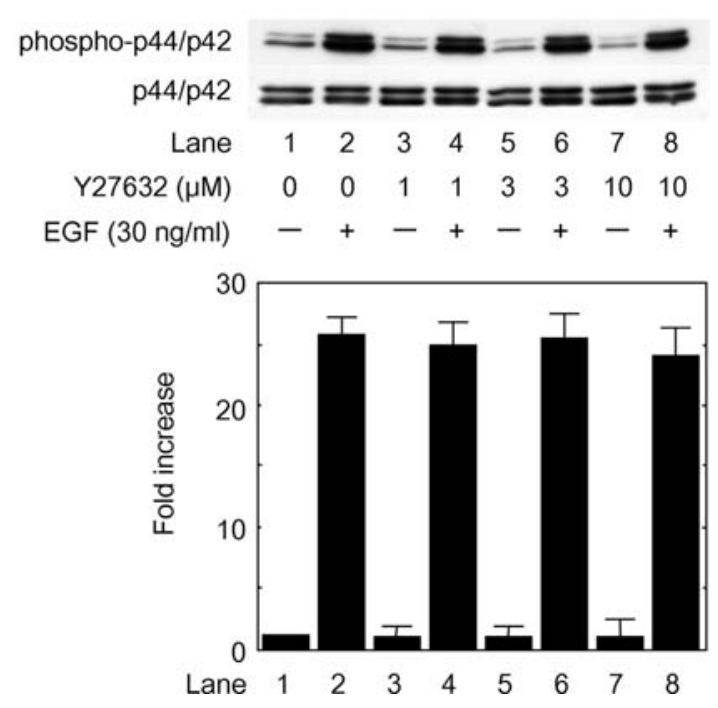

(B)
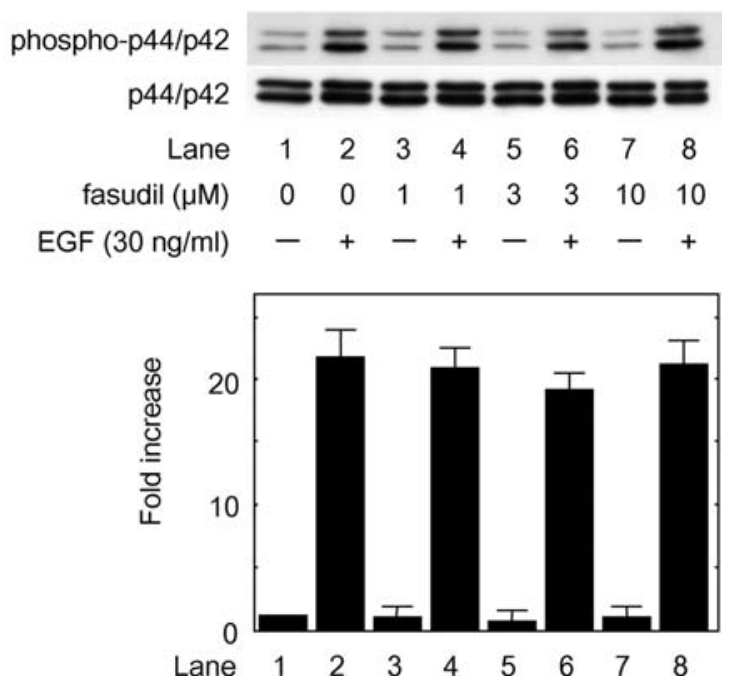

Figure 3. Effects of Rho-kinase inhibitors on EGF-induced phosphorylation of p44/p42 MAP kinase in SW480 colon cancer cells. The cells were pretreated with the indicated doses of Y27632 (A) or fasudil (B) for $60 \mathrm{~min}$, and then stimulated with $30 \mathrm{ng} / \mathrm{ml}$ EGF or vehicle for $20 \mathrm{~min}$. The extracts of cells were subjected to SDS-PAGE with subsequent Western blot analysis with antibodies against phospho-specific p44/p42 MAP kinase or p44/p42 MAP kinase. The lower bar graph shows the quantification data for the relative levels of phospho-p44/p42 MAP kinase, after normalization with respect to total p44/p42 MAP kinase, as determined by densitometry.

effect of the blockade of EGF stimulation utilizing antiEGFR-neutralizing antibodies on cell proliferation in SW480 cells grown in medium containing 3\% FCS. As expected, when the cells were treated with anti-EGFR-neutralizing antibodies for 5 days, cell growth was significantly suppressed, as compared with the cells treated with normal mouse-IgG (Fig. 1C). We also obtained similar results when the cells were cultured in medium containing $10 \%$ FCS (data not shown). Since medium containing 3\% FCS is recognized to contain various types of growth factors including EGF, it is most likely that EGF stimulation plays an important role in cell proliferation in SW480 cells. Moreover, these results led us to further investigate the role of Rho-kinase in EGFtreated colon cancer cells.

Effects of EGF on the phosphorylation of MYPT-1 in SW480 colon cancer cells. MYPT-1, which is a component of myosin phosphatase, is well known as a downstream substrate of Rho-kinase (5). In order to clarify whether EGF activates Rho-kinase in SW480 colon cancer cells, we examined the effect of EGF on the phosphorylation of MYPT-1. EGF markedly induced the phosphorylation of MYPT-1 in a timedependent manner (Fig. 2A). The effect of EGF on the phosphorylation of MYPT-1 reached its maximum at $10 \mathrm{~min}$, sustained up to $30 \mathrm{~min}$, and decreased thereafter. We next confirmed whether Y27632 suppresses the EGF-induced phosphorylation levels of MYPT-1. Y27632 (1 $\mu \mathrm{M})$ partially attenuated (Fig. 2B, lane 4 compared to lane 2) and $10 \mu \mathrm{M}$ of Y27632 almost completely suppressed the EGF-induced phosphorylation levels of MYPT-1 (Fig. 2B, lane 8). Interestingly, we observed that the basal level of MYPT-1 phosphorylation in unstimulated cells (Fig. 2B, lane 1) was also suppressed by pretreatment with Y27632 (Fig. 2B, lanes 5 and 7 compared to lane 1). Similarly, fasudil, another inhibitor of Rho-kinase (15), also attenuated the EGFinduced phosphorylation levels of MYPT-1 (Fig. 2C).
Effects of EGF on the phosphorylation of MLC in SW480 colon cancer cells. Rho is implicated in the cytoskeletal responses to extracellular signals lysophosphatidic acid and certain growth factors, which form stress fibers and cause focal adhesion $(16,17)$. It has been shown that Rho-kinase phosphorylates the MLC of smooth muscle (18), indicating that phosphorylation of MLC reflects Rho-kinase activity. To further confirm whether EGF activates Rho-kinase in SW480 cells, we examined the effect of EGF on the phosphorylation of MLC in an immunofluorescence study. When the cells were stimulated by $30 \mathrm{ng} / \mathrm{ml}$ of EGF for $20 \mathrm{~min}$, phosphorylated MLC levels were clearly strengthened in cells (Fig. 2D, panel 2 compared to panel 1). The basal level of MLC phosphorylation was suppressed by $10 \mu \mathrm{M}$ of $\mathrm{Y} 27632$ (Fig. 2D, panel 3). Moreover, pretreatment with Y27632 markedly reduced EGF-induced MLC phosphorylation (Fig. 2D, panel 4). Taken together with the results shown in Fig. 2B and C, we strongly suggest that EGF induces activation of Rho-kinase in SW480 colon cancer cells.

Effects of Y27632 or fasudil on the EGF-induced phosphorylation of p44/p42 MAP kinase in SW480 colon cancer cells. It is generally recognized that p44/p42 MAP kinase is a central element used by mammalian cells to transduce the proliferative message of a variety of growth factors (19). We therefore examined the effect of EGF on the phosphorylation of p44/p42 MAP kinase in SW480 cells. We found that EGF markedly induced the phosphorylation of p44/p42 MAP kinase in a time-dependent manner (data not shown) as previously shown in colon cancer cells (20). We next examined the effect of Y27632 or fasudil on the EGFinduced phosphorylation of p44/p42 MAP kinase. However, neither Y27632 nor fasudil affected the EGF-induced phosphorylation of $\mathrm{p} 44 / \mathrm{p} 42$ MAP kinase in the range between 1 and $10 \mu \mathrm{M}$ (Fig. 3A and B, upper panel, respectively). Therefore, it seems unlikely that Rho-kinase is 
(A)
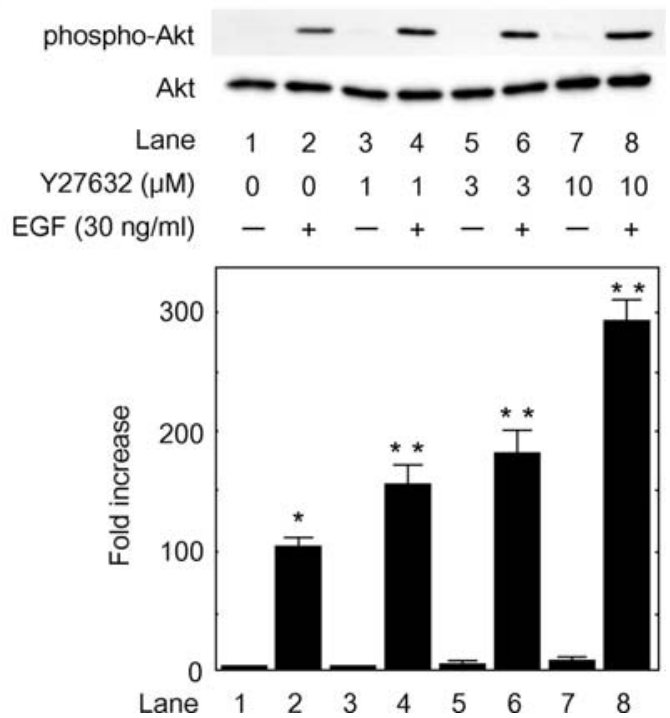

(B)
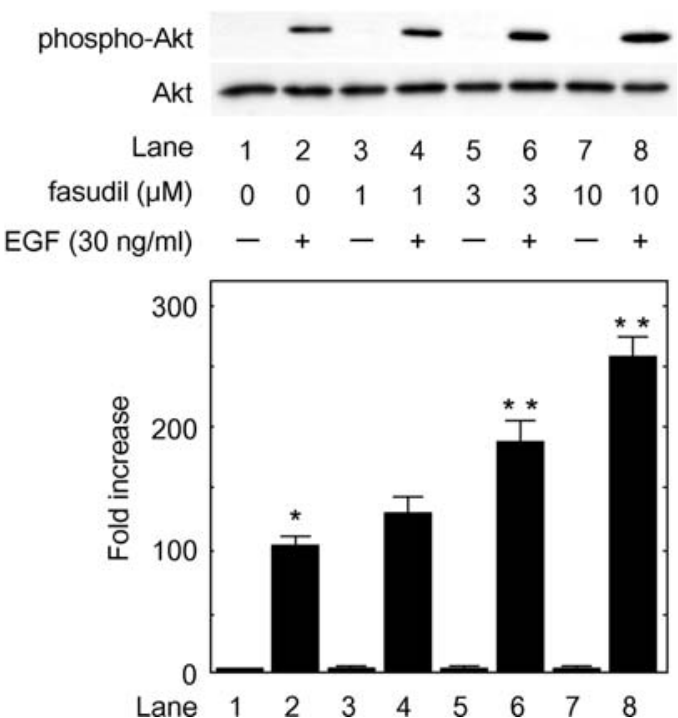

Figure 4. Effects of Rho-kinase inhibitors on EGF-induced phosphorylation of Akt in SW480 colon cancer cells. The cells were pretreated with the indicated doses of Y27632 (A) or fasudil (B) for $60 \mathrm{~min}$, and then stimulated with $30 \mathrm{ng} / \mathrm{ml}$ EGF or vehicle for 20 min. The extracts of cells were subjected to SDSPAGE with subsequent Western blot analysis with antibodies against phospho-specific Akt or Akt. The lower bar graph shows the quantification data for the relative levels of phospho-Akt, after normalization with respect to total Akt, as determined by densitometry. (*) indicates significant increase (p<0.05), as compared with the control (lane 1), respectively. (**) indicates significant increase ( $<<0.05)$, as compared with the control (lane 2 ), respectively.

involved in EGF-induced phosphorylation of p44/p42 MAP kinase.

Effects of Y27632 or fasudil on the EGF-induced phosphorylation of Akt in SW480 colon cancer cells. It is well-known that Akt regulates multiple biological processes including survival, proliferation and cell growth (21). In general, Akt promotes cell survival by inhibiting apoptosis by phosphorylating and inactivating several targets, including Bad (22), forkhead transcription factors (23) and c-Raf (24). We found that EGF markedly induced the phosphorylation of Akt in a time-dependent manner (data not shown) as previously shown in colon cancer cells (25). We next examined the effect of Y27632 or fasudil on the EGF-induced phosphorylation of Akt. Surprisingly, EGF-induced Akt phosphorylation was markedly enhanced when the cells were pretreated with Y27632, which alone failed to affect the Akt phosphorylation (Fig. 4A, upper panel). Pretreatment with $1 \mu \mathrm{M}$ of Y27632 caused $\sim 60 \%$ increase (Fig. 4A, upper panel, lane 4 compared to lane 2) and $10 \mu \mathrm{M}$ of $\mathrm{Y} 27632$ caused $300 \%$ increase (Fig. 4A, upper panel, lane 8 compared to lane 2) in the Akt phosphorylation level. In a similar way, when the cells were pretreated with fasudil, the phosphorylation level of Akt was also dose-dependently enhanced in the range between 3 , and $10 \mu \mathrm{M}$ (Fig. 4B, upper panel).

Effects of Y27632 or fasudil on the EGF-induced phosphorylation of GSK-3ß in SW480 colon cancer cells. GSK-3ß is a critical downstream element of the PI3K/Akt cell survival pathway, and its activity can be inhibited by Akt-mediated phosphorylation of GSK-3ß $(9,26)$. Therefore, we next examined the effect of Y27632 or fasudil on phosphorylation of GSK-3ß. We found that EGF markedly induced the phosphorylation of GSK-3ß in a time-dependent manner (data not shown) as reported previously (27). Y27632 increased the EGF-induced phosphorylation level of GSK-3B (Fig. 5A). Pretreatment with $3 \mu \mathrm{M}$ of $\mathrm{Y} 27632$ caused $\sim 120 \%$ increase (Fig. 5A, upper panel, lane 6 compared to lane 2) and $10 \mu \mathrm{M}$ of Y27632 caused $250 \%$ increase (Fig. 5A, upper panel, lane 8 compared to lane 2) in the GSK-3ß phosphorylation level. In addition, when the cells were pretreated with fasudil, GSK-3ß phosphorylation level was also dose-dependently enhanced in the range between 1 and $10 \mu \mathrm{M}$ (Fig. 5B, upper panel). Interestingly, unlike Akt, when the cells were pretreated with Rho-kinase inhibitors, the phosphorylation levels of GSK-3ß were increased even in EGF-unstimulated cells (Fig. 5A and B, lanes 3, 5 and 7 compared to lane 1, respectively).

Effects of Y27632 or fasudil on the cyclin D1 expression level and $R b$ phosphorylation induced by EGF in SW480 colon cancer cells. As described above, the Akt/GSK-3ß signaling pathway is closely related to the expression of cyclin D1 and phosphorylation of $\mathrm{Rb}$ (28). We next examined the effect of EGF on cyclin D1 expression level and Rb phosphorylation in SW480 cells. As shown in Fig. 6, EGF caused significant increase in both of cyclin D1 expression level and phosphorylation level of $\mathrm{Rb}$ (Fig. 6A, B and C, lane 2 compared to lane 1, respectively). Interestingly, pretreatment with Y27632 significantly enhanced the EGF-induced cyclin D1 expression level at a dose of $10 \mu \mathrm{M}$ (Fig. 6A, upper panel; 6B, lane 6 compared to lane 2). Concurrently, phosphorylation level of $\mathrm{Rb}$ was also enhanced in the cells pretreated with Y27632 (Fig. 6A, middle panel; 6C, lane 6 compared to lane 2). Moreover, phosphorylation level of $\mathrm{Rb}$ as well as cyclin D1 expression level was increased even in EGF-unstimulated cells when the cells were pretreated with Y27632 (Fig. 6A, B and $\mathrm{C}$, lanes 3 and 5 compared to lane 1), indicating that 
(A)
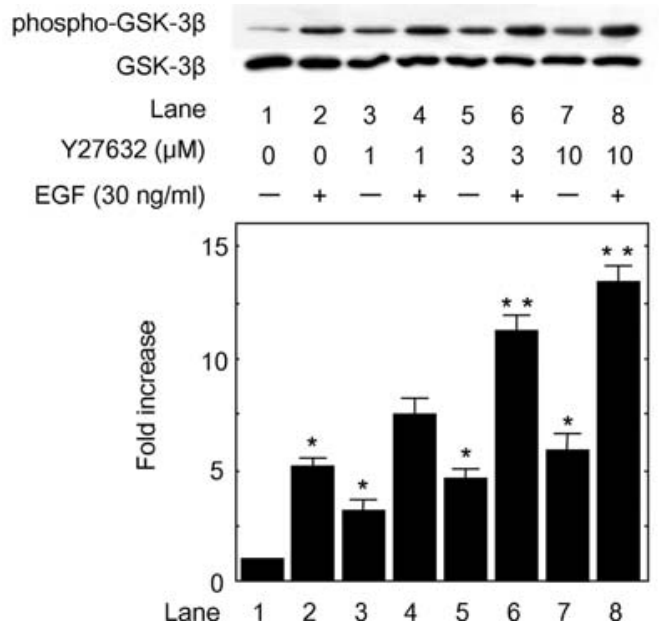

(B)
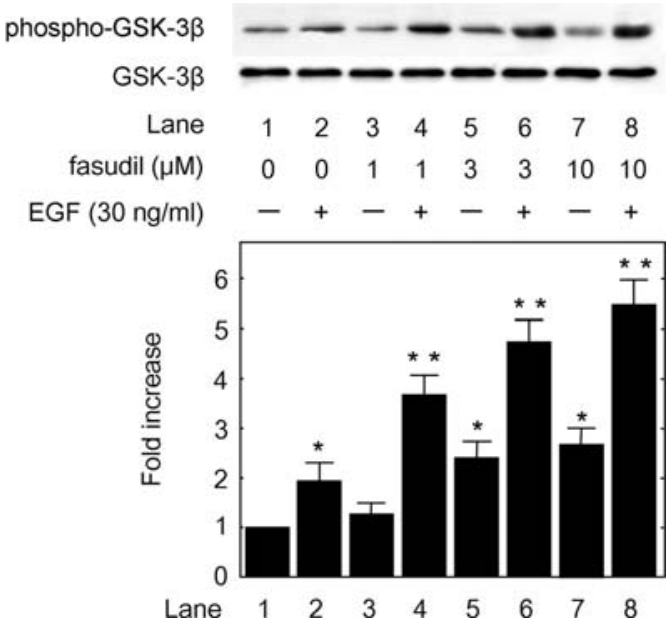

Figure 5. Effects of Rho-kinase inhibitors on EGF-induced phosphorylation of GSK-3B in SW480 colon cancer cells. The cells were pretreated with the indicated doses of Y27632 (A) or fasudil (B) for $60 \mathrm{~min}$, and then stimulated with $30 \mathrm{ng} / \mathrm{ml}$ EGF or vehicle for 20 min. The extracts of cells were subjected to SDS-PAGE with subsequent Western blot analysis with antibodies against phospho-specific GSK-3ß or GSK-3ß. The lower bar graph shows the quantification data for the relative levels of phospho-GSK-3ß, after normalization with respect to total GSK-3ß, as determined by densitometry. (*) indicates significant increase $(\mathrm{p}<0.05)$, as compared with the control (lane 1), respectively. $\left.{ }^{* *}\right)$ indicates significant increase $(\mathrm{p}<0.05)$, as compared with the control (lane 2), respectively.

(A)

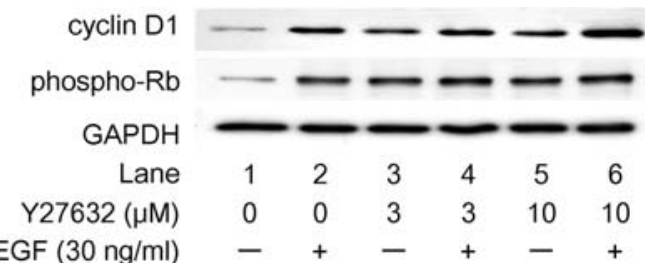

(B)

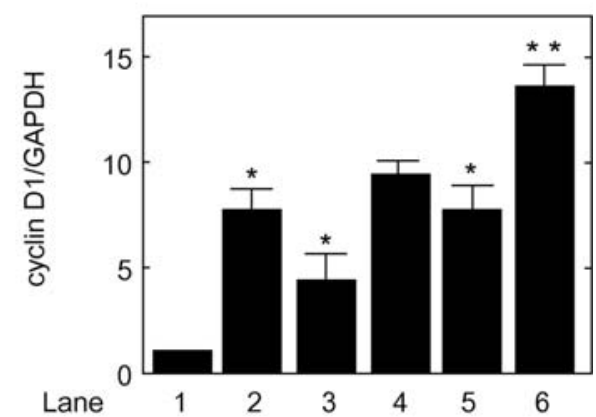

(C)

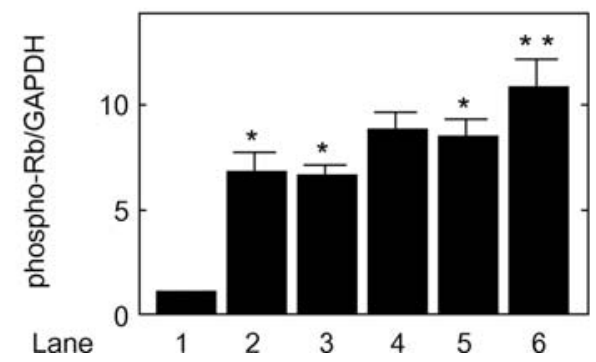

Figure 6. Effects of Rho-kinase inhibitors on EGF-induced expression of cyclin D1 and phosphorylation of Rb in SW480 colon cancer cells. (A) SW480 cells were pretreated with the indicated doses of Y27632 for $60 \mathrm{~min}$, and then stimulated with $30 \mathrm{ng} / \mathrm{ml}$ EGF or vehicle for $24 \mathrm{~h}$. The extracts of cells were subjected to SDS-PAGE with subsequent Western blot analysis with antibodies against cyclin D1, phospho-specific Rb and GAPDH. (B and C) The bar graph shows the quantification data for the relative levels of cyclin D1 (B) and phospho-Rb (C), respectively, after normalization with respect to GAPDH, as determined by densitometry. (*) indicates significant increase $(\mathrm{p}<0.05)$, as compared with the control (lane 1$)$, respectively. $(* *)$ indicates significant increase $(\mathrm{p}<0.05)$ between the indicated pairs, respectively.

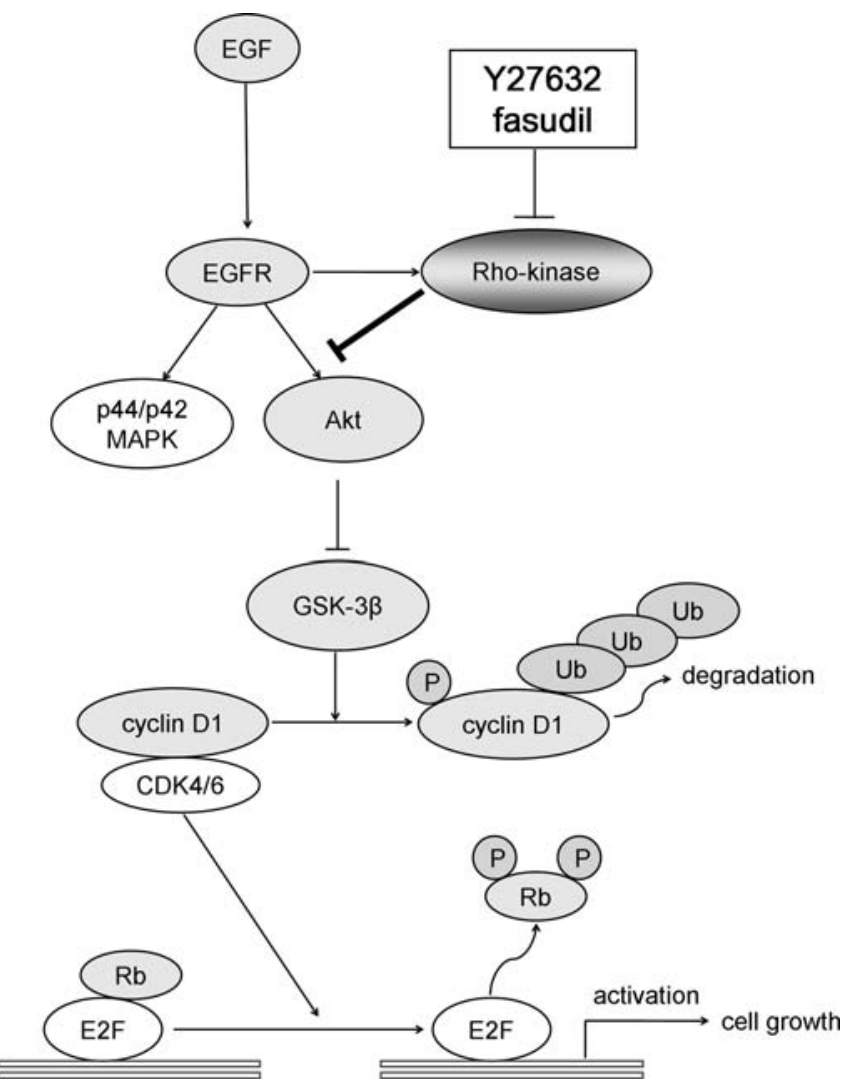

Figure 7. Schematic representation of the involvement of Rho-kinase in the mechanism of EGF-induced cell proliferation in SW480 colon cancer cells. Whereas EGF induces the activation of Rho-kinase as well as p44/p42 MAP kinase and Akt/GSK-3B, Rho-kinase also functions upstream of Akt/GSK$3 \beta$. Since GSK-3ß is typically active in unstimulated cells, inactivated GSK$3 \beta$ stimulated by EGF results in the increased expression level of cyclin D1 followed by Rb phosphorylation, leading to cell growth. Taken together, our results suggest that Rho-kinase regulates negatively EGF-induced cell proliferation in SW480 colon cancer cells. EGF, epidermal growth factor; EGFR, EGF receptor; MAPK, mitogen-activated protein kinase; GSK-3ß, glycogen synthese kinase-3B; CDK, cyclin-dependent kinase; Rb, retinoblastoma tumor suppressor protein; Ub, ubiquitination. 
Y27632 also suppressed the basal activity of Rho-kinase in SW480 colon cancer cells, which is consistent with the results shown in Fig. 2B. Since it is well known that cyclin D1 complex with CDK4/6 causes the phosphorylation and inactivation of $\mathrm{Rb}$ (11), resulting in cell proliferation, it is most likely that the activation of Rho-kinase negatively regulates colon cancer cell growth.

\section{Discussion}

In the present study, we investigated how Rho-kinase works on cell proliferation of colon cancer cells. First, we examined the effect of Y27632 on cell growth and found that Y27632 alone significantly enhanced cell proliferation in SW480 cells (Fig. 1A and B). In addition, utilizing anti-EGFR-neutralizing antibodies, we found that EGF stimulation is necessary for cell growth in SW480 cells. Therefore, in the following experiments, we examined the involvement of Rho-kinase in EGF signaling in SW480 colon cancer cells. EGF markedly induced the phosphorylation of MYPT-1, known as a downstream substrate of Rho-kinase (5), in a time-dependent manner (Fig. 2A) and Y27632 and fasudil, both of which are specific inhibitors of Rho-kinase (15), clearly suppressed the EGF-induced phosphorylation levels of MYPT-1 (Fig. 2B and $\mathrm{C}$ ). To confirm these above results, we examined the effect of extracellular EGF on the phosphorylation level of MLC, which is also known to be a downstream target of Rho-kinase (18), by an immunofluorescence study. We showed that EGF induced the phosphorylation of MLC and Y27632 suppressed EGF-induced phosphorylation of MLC (Fig. 2D), suggesting that EGF indeed stimulates the activation of Rho-kinase in colon cancer cells.

To further investigate the involvement of Rho-kinase in the EGF signaling, we next examined the effects of Rhokinase inhibitors on the EGF-induced activations of p44/p42 MAP kinase and Akt, both of which are major molecules of the signaling cascades in cancer cell proliferation (19). Whereas EGF caused the phosphorylation of both molecules, the phosphorylation of Akt, but not p44/p42 MAP kinase, was surprisingly enhanced when the cells were pretreated with either Y27632 or fasudil (Figs. 3 and 4). Since it is well known that PI3K acts upstream of Akt (7), we further investigated the effects of these inhibitors on activation of PI3K. However, both Y27632 and fasudil have little effect on the phosphorylation of PI3K induced by EGF (data not shown). Taken together, it is most likely that Rho-kinase functions at a point between Akt and PI3K in the EGF signaling, and negatively regulates its signaling in SW480 colon cancer cells.

We also demonstrated that the phosphorylation of GSK-3ß induced by EGF was markedly enhanced when the cells were pretreated with Y27632 and fasudil (Fig. 5). GSK-3ß regulates cyclin D1 proteolysis and subcellular localization, and cyclin D1 complex with CDK4 and CDK6 to stimulate their kinase activities, which in turn cause the phosphorylation and inactivation of $\mathrm{Rb}$, directing these cells toward proliferation (11). Whereas EGF markedly increased the phosphorylation of $\mathrm{Rb}$ as well as cyclin $\mathrm{D} 1$ protein expression level, pretreatment with Y27632 accelerated them (Fig. 6A), fasudil also enhanced the phosphorylation (data not shown). Taking these findings into account as a whole, our present study suggests that Rho-kinase acts as a negative regulator on colon cancer cell proliferation upstream of Akt/GSK-3ß in the EGF signaling pathway. The potential role of Rho-kinase on EGF-stimulated proliferation of colon cancer cells shown here is summarized in Fig. 7.

Accumulating evidence shows a pivotal role of the Rho/ Rho-kinase pathway in the regulator of cytoskeletal dynamics, cell adhesion and cell migration (29), indicating its central role in cancer cell invasion and metastasis. Y27632 reportedly interferes with Ras-mediated transformation, and constitutively active mutants of Rho-kinase, collaborate with activated Raf in transformation assays in NIH3T3 cells (30). On the contrary, it has been shown that inactivation of the Rho/Rho-kinase pathway promotes oncogenic Ras-induced transformation in Rat1 fibroblast cells, suggesting a negative role of Rho-kinase in oncogenic cells that is consistent with our present study (31). Thus, since the precise role of Rhokinase in cancer progression still remains controversial, further investigation is required to elucidate the exact role of Rho-kinase in colon cancer cell growth.

In conclusion, Rho-kinase regulates negatively EGFinduced cell proliferation upstream of Akt/GSK-3ß in colon cancer cells. This is the first report, which shows that Rhokinase is involved in negative regulation for EGF-induced cell proliferation, thus indicating important insights into therapeutics for colon cancer. Regulation of Rho-kinase might be considered as a new clinical target for colon cancer patients.

\section{References}

1. Bar-Sagi D and Hall A: Ras and Rho GTPases: a family reunion. Cell 103: 227-238, 2000.

2. Salhia B, Rutten F, Nakada M, Beaudry C, Berens M, Kwan A and Rutka JT: Inhibition of Rho-kinase affects astrocytoma morphology, motility, and invasion through activation of Rac1. Cancer Res 65: 8792-8800, 2005.

3. Sahai E and Marshall CJ: RHO-GTPases and cancer. Nat Rev Cancer 2: 133-142, 2002.

4. Fritz G, Just I and Kaina B: Rho GTPases are over-expressed in human tumors. Int J Cancer 81: 682-687, 1999.

5. Riento K and Ridley AJ: Rocks: multifunctional kinases in cell behaviour. Nat Rev Mol Cell Biol 4: 446-456, 2003.

6. Itoh K, Yoshioka K, Akedo H, Uehata M, Ishizaki T and Narumiya S: An essential part for Rho-associated kinase in the transcellular invasion of tumor cells. Nat Med 5: 221-225, 1999.

7. Henson ES and Gibson SB: Surviving cell death through epidermal growth factor (EGF) signal transduction pathways: implications for cancer therapy. Cell Signal 18: 2089-2097, 2006.

8. Sutherland C, Leighton IA and Cohen P: Inactivation of glycogen synthase kinase- 3 beta by phosphorylation: new kinase connections in insulin and growth-factor signalling. Biochem J 296: 15-19, 1993.

9. Cross DA, Alessi DR, Cohen P, Andjelkovich M and Hemmings B: A. Inhibition of glycogen synthase kinase-3 by insulin mediated by protein kinase B. Nature 378: 785-789, 1995.

10. Force T and Woodgett JR: Unique and overlapping functions of GSK-3 isoforms in cell differentiation and proliferation and cardiovascular development. J Biol Chem 284: 9643-9647, 2009.

11. Diehl JA, Cheng M, Roussel MF and Sherr CJ: Glycogen synthase kinase-3beta regulates cyclin D1 proteolysis and subcellular localization. Genes Dev 12: 3499-3511, 1998.

12. Takahashi-Yanaga F and Sasaguri T: GSK-3ß regulates cyclin D1 expression: a new target for chemotherapy. Cell Signal 20: 581-589, 2008. 
13. Adachi S, Nagao T, To S, Joe AK, Shimizu M, MatsushimaNishiwaki R, Kozawa O, Moriwaki H, Maxfield FR and Weinstein IB: (-)-Epigallocatechin gallate causes internalization of the epidermal growth factor receptor in human colon cancer cells. Carcinogenesis 29: 1986-1993, 2008.

14. Adachi S, Natsume H, Yamauchi, J, Matsushima-Nishiwaki R, Joe AK, Moriwaki H and Kozawa O: p38 MAP kinase controls EGF receptor downregulation via phosphorylation at Ser1046/ 1047. Cancer Lett 277: 108-113, 2009.

15. Shimokawa H and Rashid M: Development of Rho-kinase inhibitors for cardiovascular medicine. Trends Pharmacol Sci 28: 296-302, 2007

16. Ridley AJ and Hall A: The small GTP-binding protein rho regulates the assembly of focal adhesions and actin stress fibers in response to growth factors. Cell 70: 389-399, 1992.

17. Ridley AJ and Hall A: Signal transduction pathways regulating Rho-mediated stress fibre formation: requirement for a tyrosine kinase. EMBO J 13: 2600-2610, 1994.

18. Amano M, Ito M, Kimura K, Fukata Y, Chihara K, Nakano T, Matsuura Y and Kaibuchi K: Phosphorylation and activation of myosin by Rho-associated kinase (Rho-kinase). J Biol Chem 271: 20246-20249, 1996.

19. Kyriakis JM and Avruch J: Mammalian mitogen-activated protein kinase signal transduction pathways activated by stress and inflammation. Physiol Rev 81: 807-869, 2001.

20. Rice PL, Washington M, Schleman S, Beard KS, Driggers LJ and Ahnen DJ: Sulindac sulfide inhibits epidermal growth factorinduced phosphorylation of extracellular-regulated kinase $1 / 2$ and Bad in human colon cancer cells. Cancer Res 63: 616-620, 2003.

21. Cheng JQ, Lindsley CW, Cheng GZ, Yang H and Nicosia SV: The Akt/PKB pathway: molecular target for cancer drug discovery. Oncogene 24: 7482-7492, 2005.

22. Cardone MH, Roy N, Stennicke HR, Salvesen GS, Franke TF Stanbridge E, Frisch S and Reed JC: Regulation of cell death protease caspase-9 by phosphorylation. Science 282: 1318-1321, 1998.
23. Brunet A, Bonni A, Zigmond MJ, Lin MZ, Juo P, Hu LS, Anderson MJ, Arden KC, Blenis J and Greenberg ME: Akt promotes cell survival by phosphorylating and inhibiting a Forkhead transcription factor. Cell 96: 857-868, 1999.

24. Zimmermann S and Moelling K: Phosphorylation and regulation of Raf by Akt (protein kinase B). Science 286: 1741$1744,1999$.

25. Bishnupuri KS, Luo Q, Murmu N, Houchen CW, Anant S and Dieckgraefe BK: Reg IV activates the epidermal growth factor receptor/Akt/AP-1 signaling pathway in colon adenocarcinomas. Gastroenterology 130: 137-149, 2006.

26. Srivastava AK and Pandey SK: Potential mechanism(s) involved in the regulation of glycogen synthesis by insulin. Mol Cell Biochem 182: 135-141, 1998.

27. Jaszewski R, Millar B, Hatfield JS, Nogothu K, Finkenauer R, Rishi AK, Naumoff JA, Kucuk O, Axelrod BN and Majumdar AP: Folic acid reduces nuclear translocation of beta-catenin in rectal mucosal crypts of patients with colorectal adenomas. Cancer Lett 206: 27-33, 2004.

28. Pruitt K and Der CJ: Ras and Rho regulation of the cell cycle and oncogenesis. Cancer Lett 171: 1-10, 2001.

29. Jaffe $A B$ and Hall A: Rho GTPases: biochemistry and biology. Annu Rev Cell Dev Biol 21: 247-269, 2005.

30. Sahai E, Ishizaki T, Narumiya $\mathrm{S}$ and Treisman R: Transformation mediated by RhoA requires activity of ROCK kinases. Curr Biol 9: 136-145, 1999.

31. Izawa I, Amano M, Chihara K, Yamamoto T and Kaibuchi K: Possible involvement of the inactivation of the Rho-Rho-kinase pathway in oncogenic Ras-induced transformation. Oncogene 17: 2863-2871, 1998. 